\title{
Generation of an octave-spanning supercontinuum in highly nonlinear fibers pumped by noise-like pulses \\ Shih-Shian Lin ${ }^{\mathrm{a}}$, Sheng-Kwang Hwang ${ }^{\mathrm{b}, \mathrm{c}}$, Jia-Ming Liu *a,d \\ anstitute of Photonic System, National Chiao Tung University, Tainan, Taiwan; \\ ${ }^{b}$ Department of Photonics, National Cheng Kung University, Tainan, Taiwan; \\ ${ }^{c}$ Advanced Optoelectronic Technology Center, National Cheng Kung University, Tainan, Taiwan; \\ ${ }^{\mathrm{d}}$ Electrical Engineering Department, University of California, Los Angeles, California, USA
}

\begin{abstract}
A supercontinuum generation system is developed, which consists of an erbium-doped fiber ring laser, an erbium-doped fiber amplifier, and a 100-m highly nonlinear fiber. Through nonlinear polarization rotation, the fiber ring laser generates a train of noise-like pulses in the form of repetitive picosecond pulse packets consisting of femtosecond noise-like fine temporal structures. The noise-like pulses are amplified before being sent into the highly nonlinear fiber. As a result, an octave-spanning supercontinuum from $1177 \mathrm{~nm}$ to $2449 \mathrm{~nm}$ is obtained, which has a 20-dB spectral width of $980 \mathrm{~nm}$. Because of the nonlinearity of the fiber amplifier, the duration of the noise-like pulses is shortened while their average power is enhanced. However, the enhanced pulse energy makes the key contribution to the spectral broadening of the resulting spuercontinuum in this study since the highly nonlinear fiber is so long that the effect of the pulse compression on supercontinuum generation is weak.
\end{abstract}

Keywords: pulse lasers, fiber lasers, mode-locked lasers, ultrafast lasers, supercontinuum generation, nonlinear optics, pulse amplifier, fiber amplifier, noise-like pulses, femtosecond phenomena.

\section{INTRODUCTION}

Supercontinuum sources, owing to their numerous applications in such areas as microscopy [1], spectroscopy [2], and optical communication [3], have been the object of extensive investigation over the past few decades [4]. Unlike traditional broadband light sources, such as mercury lamps, supercontinnuum possesses the characteristics of single mode, broad and flat spectrum, and high temporal coherence. However, for certain applications, such as optical coherence tomography, optical sensing [5,6], and optical communications [7], broadband light sources with low temporal coherence are preferred. Therefore, some recent studies have been devoted to the investigation of supercontinuum generation using noise-like pulses [8-13]. The noise-like pulses were composed of sub-nanosecond wavepackets with a fine inner structure of subpicosecond pulses that have stochastically changing durations and peak intensities [14]. Such a unique characteristic of the noise-like pulses leads to a broad spectrum of low temporal coherence, which can therefore be used as pump pulses for the generation of supercontimuum of low temporal coherence.

Different approaches have been proposed for supercontinuum generation using noise-like pulses. For example, in one approach a highly nonlinear fiber was inserted inside a laser ring cavity operating at high pump power levels [15]. As a result, the Raman scatting was considerably excited and a spectrum of 135-nm width is obtained [16]. In another approach a highly nonlinear fiber was placed outside the laser cavity instead and pumped by the noise-like pulses from the laser. A spectral width spanning over several hundreds of nanometers was achieved $[8,9]$. To increase the spectral width of supercontinuum, yet another approach was to strongly amplify the noise-like pulses before they were launched into a highly nonlinear fiber [10]. As a result, a supercontinuum spectrum spanning from 1208 to $2111 \mathrm{~nm}$ was obtained using a 1-m nonlinear fiber pumped by amplified noise-like pulses. In this study, we demonstrate the generation of an octave-spanning supercontinuum from $1177 \mathrm{~nm}$ to $2449 \mathrm{~nm}$ using a 100-m highly nonlinear fiber pumped by amplified noise-like pulses.

*liu@seas.ucla.edu

CCC code: $0277-786 \mathrm{X} / 14 / \$ 18 \cdot$ doi: $10.1117 / 12.2061258$ 


\section{EXPERIMENTAL RESULTS AND DISCUSSION}

\subsection{Noise-like pulse generation}

Figure 1 shows the experimental arrangement for the all-fiber supercontinuum generation system used in this study. The ring cavity consisted of a $0.6-\mathrm{m}$ erbium-doped fiber with a peak absorption coefficient of $80 \mathrm{~dB} / \mathrm{m}$ at $1530 \mathrm{~nm}$. The erbium-doped fiber was pumped by a pump laser diode delivering a power of $480 \mathrm{~mW}$ at $976 \mathrm{~nm}$. The cavity also included a 3-m dispersion compensation fiber with a group velocity dispersion coefficient of $-97.7 \mathrm{ps} / \mathrm{km}-\mathrm{nm}$ at 1550 $\mathrm{nm}$, a polarization dependent isolator to ensure the unidirectional propagation of optical signals but also to limit their polarization direction, a 10/90 coupler to deliver the pulse train out of the cavity, and two polarization controllers to adjust the polarization inside the cavity. The total length of the ring cavity was $12.7 \mathrm{~m}$. By appropriately selecting the orientations of the two polarization controllers, the laser oscillator was able to steadily generate noise-like pulses in the form of repetitive picosecond pulse packets consisting of femtosecond noise-like fine temporal structures. The fundamental repetition rate of the picosecond pulse packets was $15.5 \mathrm{MHz}$, which indicates that the oscillator generated with one pulse packet per round trip. The average output power from the $10 \%$ output port of the coupler was $14 \mathrm{~mW}$, and the pulse energy was $0.9 \mathrm{~nJ}$. The optical spectrum had a peak wavelength at around $1560.9 \mathrm{~nm}$ and a 3-dB spectral width of $13.1 \mathrm{~nm}$. The autocorrelation traces of the laser output are shown in Figures 2(a) and (b), from which the pedestal duration and the spike duration were found to be $44.4 \mathrm{ps}$ and $352 \mathrm{fs}$, respectively. The ratio between the spike peak and the shoulder level of the autocorrelation trace in Figure 2(a) is around 2, which suggests a characteristic of noise-like pulses.

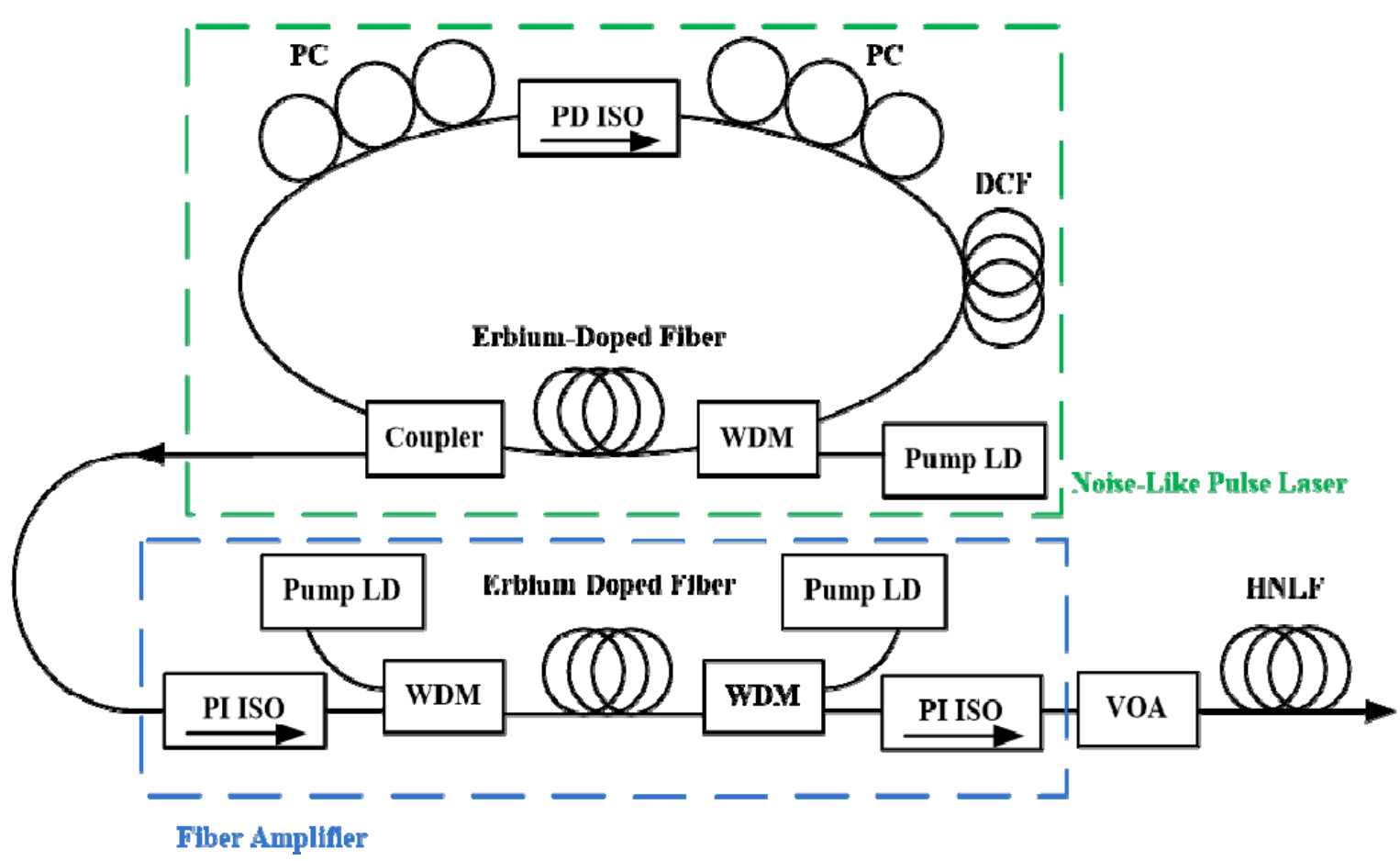

Figure 1. Experimental setup of supercontinuum generation. DCF, dispersion compensation fiber; PC, polarization controller; PD ISO, polarization-dependent isolator; PI ISO, polarization-independent isolator; Pump LD, pump laser diode; HNLF, highly nonlinear fiber; VOA, variable optical attenuator; WDM, wavelength division multiplexer. 


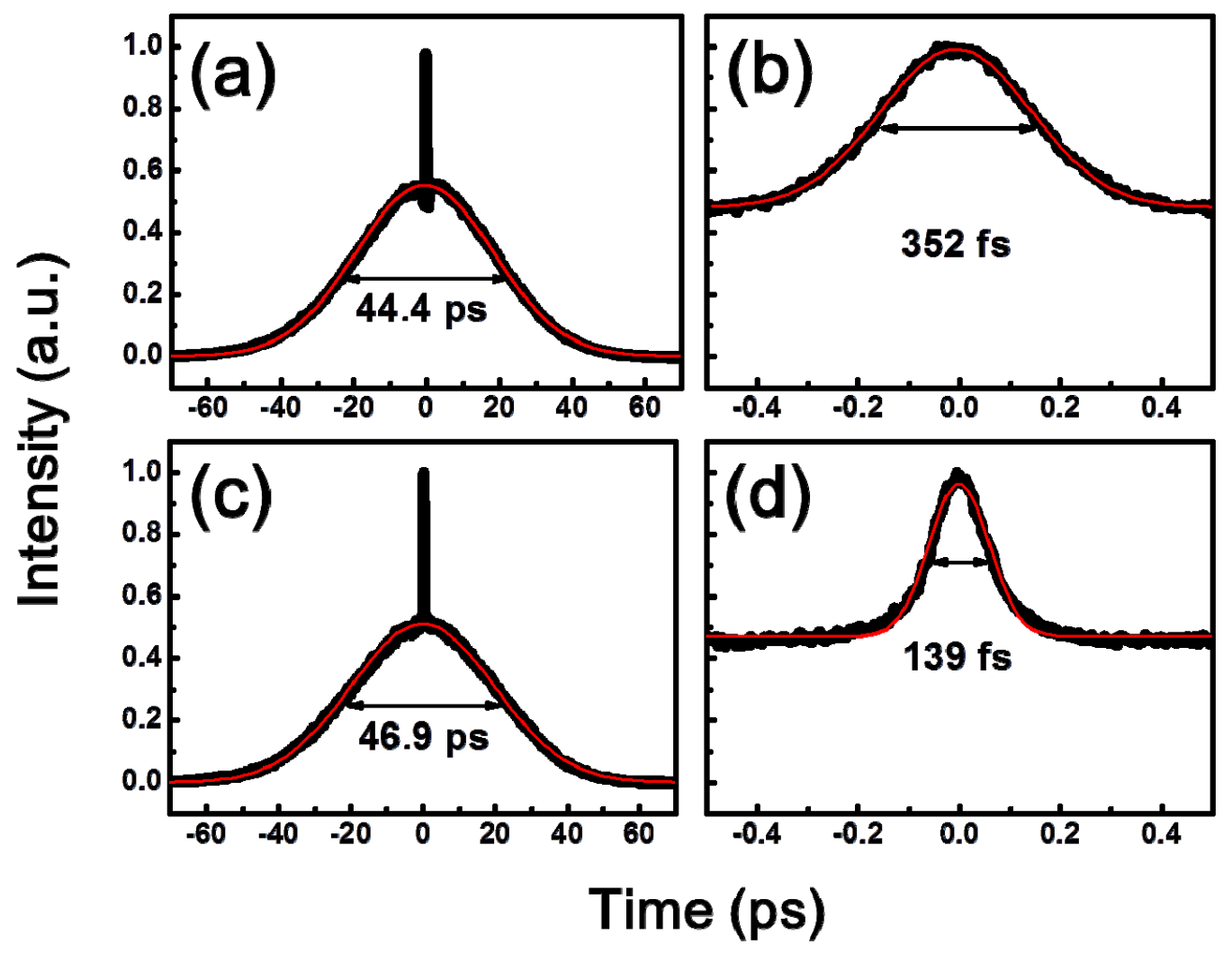

Figure 2. Autocorrelation trace of noise-like pulses before amplification (a) and after amplification (c). (b) and (d) are magnification of the spikes in (a) and (c), respectively. Red curves are Gaussian fitting curves.

\subsection{Amplification of noise-like pulses}

To increase the average power of the noise-like pulses, the laser output was sent through an erbium-doped fiber amplifier with a bi-directional pump configuration. The amplifier was composed of two 980/1550-nm wavelength-divisionmultiplexed couplers and two 976-nm pump laser diodes. To ensure unidirectional propagation of the noise-like pulses and to avoid back reflection, two polarization-independent isolators are placed at the input and output ports of the amplifier, respectively. The output power increased with the pump power, as shown in Figure 3(a), where a linear relationship with a slope of 0.19 is observed. The output average power is given as:

$$
P_{\mathrm{s}}^{\text {out }}=0.19 \times P_{\mathrm{p}}^{\text {in }}-3.39 \text {, }
$$

where $P_{\mathrm{s}}^{\text {out }}$ and $P_{\mathrm{p}}^{\text {in }}$ are the output power and the pump power, respectively. The maximum output power is $202 \mathrm{~mW}$ at a pump power level of $1088 \mathrm{~mW}$. While the average power of the noise-like pulses increased, their temporal profile also evolved with the pump power. As seen in Figure 3(b), the peak duration was significantly compressed from 378 fs to $139 \mathrm{fs}$ with the increase of the pump power; by contrast, the pedestal duration increased slightly from $42.7 \mathrm{ps}$ to $46.9 \mathrm{ps}$. For the range of the pump power under study, the peak duration and the pedestal duration could be compressed by a factor of about 0.4 and 1 , respectively. As a result, the peak power of the noise-like pulses was increased not only because of the average power enhancement but also owing to the temporal compression of the pulses. 

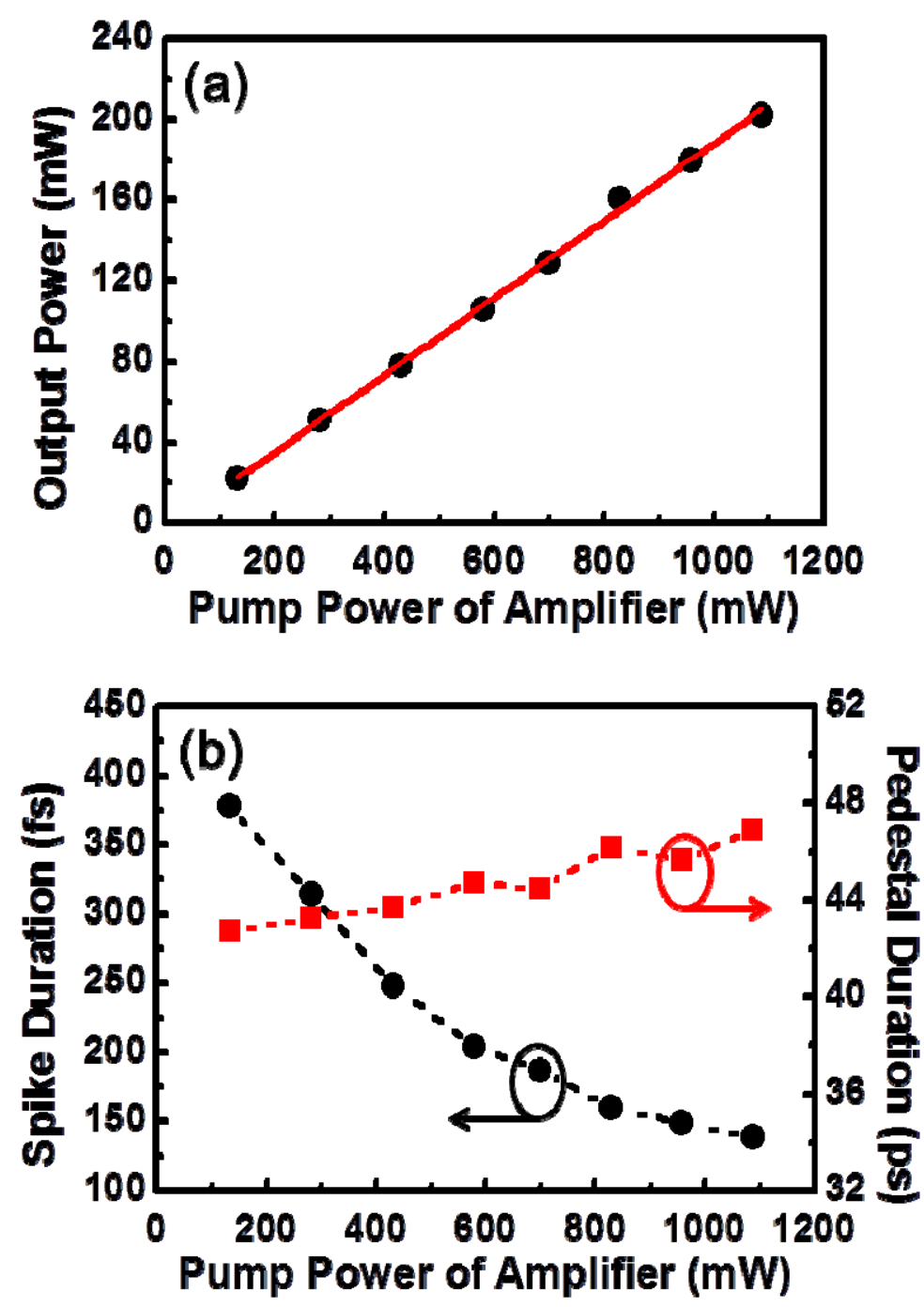

Figure 3. (a) Output power versus pump power of erbium-doped fiber amplifier. The red line represents a linear fit. (b) The spike duration and pedestal duration versus pump power of the amplifier.

\subsection{Supercontinuum generation}

The amplified noise-like pulses were launched into a 100-m-long highly nonlinear fiber. The effective area and the nonlinear coefficient of the highly nonlinear fiber were $12.5 \mu \mathrm{m}$ at $1550 \mathrm{~nm}$ and $10.7 \mathrm{~W}^{-1}-\mathrm{km}^{-1}$, respectively. Two different operating conditions were considered in this study for supercontinuum generation. First, while the pump power of the amplifier was fixed at the maximum level of $1088 \mathrm{~mW}$, the launched average power of the amplified noise-like pulses was adjusted through the variable optical attenuator shown in Figure 1. Under this condition, the spike duration in the autocorrelation trace of the amplified noise-like pulses was maintained at 139 fs throughout the launched power adjustment. Second, while the power attenuation through the variable optical attenuator is kept at the minimum level, the pump power of the amplifier was adjusted to vary the launched power of the amplified noise-like pulses. Under this condition, the spike duration decreased with the launched power. Therefore, the duration of the noise-like pulses obtained under the first operating condition was shorter than that obtained under the second operating condition.

Figure 4(a) shows the spectra of the supercontinua at four different launched powers of the amplified noise-like pulses under the two operating conditions mentioned above. First, the spectra broaden significantly with the average power of 
the noise-like pulses launched into the highly nonlinear fiber. This is more easily observed in Figure 4(b), where a 20-dB spectral width of each spectrum is defined and demonstrated. At the maximum launched power of $202 \mathrm{~mW}$, which corresponds to a pulse packet energy of $13 \mathrm{~nJ}$, an octave-spanning supercontinuum from $1177 \mathrm{~nm}$ to $2449 \mathrm{~nm}$ was obtained, which has a 20-dB spectral width of $980 \mathrm{~nm}$. Second, the spectra of the supercontinua depend negligibly on the pulse duration. As demonstrated in our previous study [10], where a highly nonlinear fiber of only 1-m long was used, a shorter duration of noise-like pulses generally generates a broader spectrum of supercontinuum under the same average launched power. Therefore, the result shown in Figure 4 indicates that the length of the highly nonlinear fiber used in this study is so long that even a noise-like pulse of a longer duration but of the same average launched power can excite a similar level of nonlinear effects to achieve a similar supercontinuum spectrum.

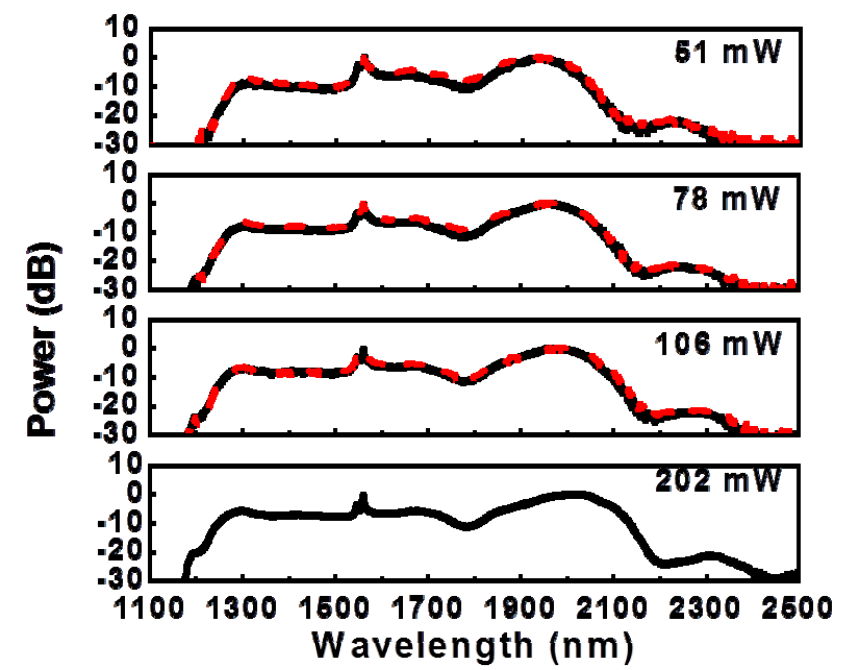

(a)

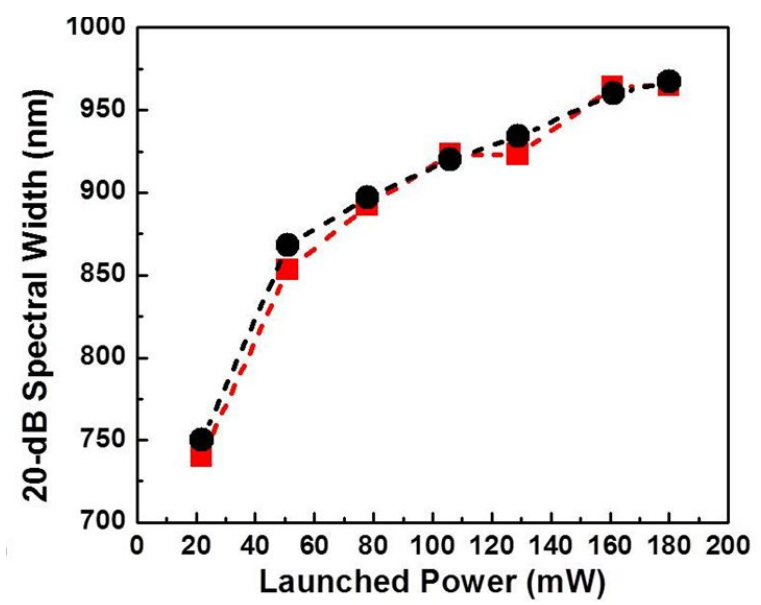

(b)

Figure 4. (a) Optical spectra and (b) 20-dB spectral widths of supercontinua at different average powers of noise-like pulses launched into the highly nonlinear fiber. Each launched power studied in (a) is indicated in each plot. Black curves and symbols were obtained when the launched power was adjusted through the variable optical attenuator, while red curves and symbols were obtained when the launched power was changed by varying the pump power of the fiber amplifier. 


\section{CONCLUSION}

By seeding amplified noise-like pulses of $13 \mathrm{~nJ}$ pulse energy into a 100-m highly nonlinear fiber, an octave-spanning supercontinuum from $1177 \mathrm{~nm}$ to $2449 \mathrm{~nm}$ for a $20-\mathrm{dB}$ spectral width of $980 \mathrm{~nm}$ was obtained. Because of the nonlinearity of the fiber amplifier, the duration of the noise-like pulses was shortened while their average power was enhanced. In this study, the enhanced pulse energy made the key contribution to the spectral broadening of the resulting spuercontinuum since the highly nonlinear fiber was so long that the effect of the pulse compression on supercontinuum generation was weak.

\section{ACKNOWLEDGEMENTS}

S.K. Hwang's work is supported by the National Science Council of Taiwan under Contract NSC102-2112-M-006-004.

\section{REFERENCES}

[1] G. Humbert, W. Wadsworth, S. Leon-Saval et al., "Supercontinuum generation system for optical coherence tomography based on tapered photonic crystal fibre," Optics Express, 14(4), 1596-1603 (2006).

[2] A. L. Dobryakov, S. A. Kovalenko, A. Weigel et al., "Femtosecond pump/supercontinuum-probe spectroscopy: Optimized setup and signal analysis for single-shot spectral referencing," Review of Scientific Instruments, 81(11), - (2010).

[3] R. Wu, V. Torres-Company, D. E. Leaird et al., "Supercontinuum-based 10-GHz flat-topped optical frequency comb generation," Optics Express, 21(5), 6045-6052 (2013).

[4] R. R. Alfano, and S. L. Shapiro, "Emission in the Region 4000 to 7000 \&Aring; Via Four-Photon Coupling in Glass," Physical Review Letters, 24(11), 584 (1970).

[5] V. Goloborodko, S. Keren, A. Rosenthal et al., "Measuring Temperature Profiles in High-Power Optical Fiber Components," Applied Optics, 42(13), 2284-2288 (2003).

[6] M. A. Putnam, M. L. Dennis, I. N. Duling Iii et al., "Broadband square-pulse operation of a passively modelocked fiber laser for fiber Bragg grating interrogation," Optics Letters, 23(2), 138-140 (1998).

[7] S. Keren, E. Brand, Y. Levi et al., "Data storage in optical fibers and reconstruction by use of low-coherence spectral interferometry," Optics Letters, 27(2), 125-127 (2002).

[8] A. Zaytsev, C.-H. Lin, Y.-J. You et al., "Supercontinuum generation by noise-like pulses transmitted through normally dispersive standard single-mode fibers," Optics Express, 21(13), 16056-16062 (2013).

[9] Y. Takushima, K. Yasunaka, Y. Ozeki et al., "87 nm bandwidth noise-like pulse generation from erbium-doped fibre laser," Electronics Letters, 41(7), 399-400 (2005).

[10] S.-S. Lin, S.-K. Hwang, and J.-M. Liu, "Supercontinuum generation in highly nonlinear fibers using amplified noise-like optical pulses," Optics Express, 22(4), 4152-4160 (2014).

[11] J. C. Hernandez-Garcia, O. Pottiez, and J. M. Estudillo-Ayala, "Supercontinuum generation in a standard fiber pumped by noise-like pulses from a figure-eight fiber laser," Laser Physics, 22(1), 221-226 (2012).

[12] J. C. Hernandez-Garcia, O. Pottiez, J. M. Estudillo-Ayala et al., "Numerical analysis of a broadband spectrum generated in a standard fiber by noise-like pulses from a passively mode-locked fiber laser," Optics Communications, 285(7), 1915-1919 (2012).

[13] L. M. Zhao, D. Y. Tang, T. H. Cheng et al., "120 nm Bandwidth noise-like pulse generation in an erbiumdoped fiber laser," Optics Communications, 281(1), 157-161 (2008).

[14] M. Horowitz, Y. Barad, and Y. Silberberg, "Noiselike pulses with a broadband spectrum generated from an erbium-doped fiber laser," Opt. Lett., 22(11), 799-801 (1997).

[15] T. North, and M. Rochette, "Raman-induced noiselike pulses in a highly nonlinear and dispersive all-fiber ring laser," Opt. Lett., 38(6), 890-892 (2013).

[16] L. A. Vazquez-Zuniga, and J. Yoonchan, "Super-Broadband Noise-Like Pulse Erbium-Doped Fiber Ring Laser With a Highly Nonlinear Fiber for Raman Gain Enhancement," Photonics Technology Letters, IEEE, 24(17), 1549-1551 (2012). 